\title{
RESEARCH
}

\section{Impact of influenza exposure on rates of hospital admissions and physician visits because of respiratory illness among pregnant women}

\author{
Linda Dodds, Shelly A. McNeil, Deshayne B. Fell, Victoria M. Allen, Ann Coombs, Jeffrey Scott, \\ Noni MacDonald
}

\section{ABSTRACT}

Background: Excess deaths have occurred among pregnant women during influenza pandemics, but the impact of influenza during nonpandemic years is unclear. We evaluated the impact of exposure during nonpandemic influenza seasons on the rates of hospital admissions and physician visits because of respiratory illness among pregnant women.

Methods: We conducted a 13-year (1990-2002) populationbased cohort study involving pregnant women in Nova Scotia. We compared rates of hospital admissions and physician office visits because of respiratory illness during the influenza season in each trimester of pregnancy with rates during the influenza season in the year before pregnancy and with rates in non-influenza seasons. Poisson regression analyses were performed to estimate rate ratios and $95 \%$ confidence intervals (Cls).

Results: Of 134188 pregnant women in the study cohort, 510 ( $0.4 \%$ ) were admitted to hospital because of a respiratory illness during pregnancy and 33775 (25.2\%) visited their physician for the same reason during pregnancy. During the influenza seasons, the rate ratio of hospital admissions in the third trimester compared with admissions in the year before pregnancy was $7.9(95 \% \mathrm{Cl} 5.0-12.5)$ among women with comorbidities and 5.1 (95\% Cl 3.6-7.3) among those without comorbidities. The rate of hospital admissions in the third trimester among women without comorbidities was 7.4 per 10000 woman-months during the influenza season, compared with 5.4 and 3.1 per 10000 woman-months during the peri- and non-influenza seasons respectively. Corresponding rates among women with comorbidities were 44.9, 9.3 and 18.9 per 10000 woman-months. Only $6.7 \%$ of women with comorbidities had received influenza immunization.

Interpretation: Our data support the recommendation that pregnant women with comorbidities should receive influenza vaccination regardless of their stage of pregnancy during the influenza season. Since hospital admissions because of respiratory illness during the influenza season were also increased among pregnant women without comorbidities, all pregnant women are likely to benefit from influenza vaccination.

CMAJ 2007; $176(4): 463-8$
I nfluenza viruses are the most common cause of serious respiratory illnesses in winter around the world. Incontrovertible evidence showing reductions of influenzaassociated morbidity and mortality with influenza immunization in high-risk groups has led to the National Advisory Committee on Immunization in Canada and the Advisory Committee on Immunization Practices in the United States to recommend annual vaccination of adults over the age of 65 years, children 6-23 months of age and all people with high-risk comorbid medical conditions..$^{1,2}$

Among previously healthy pregnant women, excess deaths were documented during the influenza pandemics of I9I8/I9 and $1957 / 58 .{ }^{3,4}$ In both pandemics, pneumonia was reported in $50 \%$ of the cases involving pregnant women and was associated with a maternal mortality rate of $50 \%$ and a high rate of pregnancy loss (52\% in 1918/19). Although fatal and near-fatal influenza has been reported in pregnant women during intra-pandemic influenza seasons, ${ }^{5-7}$ the true impact of influenza on pregnant women during nonpandemic influenza seasons is not clear. Neuzil and colleagues conducted a I9-year retrospective cohort study in a Tennessee Medicaid population and found an increased risk of hospital admission during the influenza seasons in all trimesters of pregnancy. ${ }^{8}$ On the basis of documented maternal morbidity and mortality during influenza pandemics and the data reported by Neuzil and colleagues about the increased risk of hospital admission among healthy pregnant women during nonpandemic influenza seasons, ${ }^{8}$ the US Advisory Committee on Immunization Practices expanded its recommendation for influenza immunization in 2004 to include all women who will be pregnant during an influenza season, regardless of their stage of pregnancy. ${ }^{2}$ In Canada, the National Advisory Committee on Immunization continues to recommend influenza vaccine for pregnant women if they have medical comorbidities known to place them at increased risk of influenzaassociated complications, they are a household contact of a person at high-risk or they will deliver during an influenza season and thus become a household contact of a high-risk newborn. ${ }^{1}$

Existing studies demonstrating an increased risk of hospital admission among pregnant women during influenza seasons are limited by study design and generalizability. ${ }^{5-10}$ These limitations identify a need for robust, population-based data on which to base public health policy for influenza immunization during pregnancy. We conducted a population-based study to 
determine whether the rates of hospital admissions and physician office visits because of respiratory illness among pregnant women with and without medical comorbidities differ between influenza, peri-influenza and non-influenza seasons and between these seasons and the year before pregnancy.

\section{Methods}

We conducted a population-based retrospective cohort study using the Nova Scotia Atlee Perinatal Database (NSAPD). The cohort comprised all Nova Scotia residents who delivered an infant weighing $500 \mathrm{~g}$ or more or who delivered at 20 weeks' gestation or later between Jan. I, I99o, and Dec. 3I, 2002. Information on dates of each trimester of pregnancy and on pre-existing risk factors for severe influenza (e.g., asthma and cardiovascular disease) was obtained from the NSAPD. The NSAPD contains extensive information related to maternal medical conditions, maternal risk factors and demographic factors, the prenatal period, labour and delivery factors and neonatal outcomes for all hospital deliveries among Nova Scotia residents. Data are abstracted from medical records by trained health records personnel using standardized data collection forms. An on-going data quality assurance program, which includes periodic data reabstraction studies, indicates that the data are reliable. ${ }^{11}$

Our primary outcome measures - hospital admissions and physician office visits because of respiratory illness - were determined from the Canadian Institute for Health Information's Discharge Abstract Database (hospital admissions) and the Nova Scotia Medical Services Insurance Database (physician office visits). These databases capture all uses of the formal public health care system in Nova Scotia. There is no parallel private system in the province. In addition, a master registration file tracks individuals moving into, and out of, the province by their enrolment in the Nova Scotia Health Services Program. We estimated socioeconomic status from data in the Family Benefits Database, which records receipt of financial support through assistance programs of the provincial and federal governments on a yearly basis. The administrative databases were successfully linked to the NSAPD for $96 \%$ of the women in the cohort. The individual categories of respiratory conditions and the corresponding International Classification of Diseases (ICD) codes that we used to identify hospital admissions and physician visits of interest are shown in Appendix I (available online at www.cmaj.ca/cgi /content/full/I76/4/463/DCI). The ICD codes selected were adapted from those used by Neuzil and colleagues ${ }^{8}$ except for the code for asthma and the codes for respiratory conditions related to occupational exposures. To be conservative, we chose not to include asthma as a respiratory condition because not all exacerbations of asthma are due to influenza, and any cases of asthma that are due to influenza most likely would have an accompanying influenza-related code on the record.

We obtained information on the defined periods of influenza season from the Nova Scotia Department of Health for the study years (1990-2002). For the purposes of our study, the beginning of the influenza season was defined as the time when 2 or more isolates of influenza were identified in Nova Scotia in sequential weeks or 3 or more isolates were identified in a single week. The end of the influenza season was defined as the time when no in- fluenza isolates were identified in the province in 2 or more sequential weeks unless the lapse was followed by a large number of isolates. The peri-influenza season was defined as the period between Nov. I and the start of the influenza season and the 2week period following influenza season. The non-influenza season was defined as the end of the peri-influenza season until Oct. 31. Data from the Medical Services Insurance Database for October 1999 onward include a code for influenza vaccination; therefore, after this date, we were able to examine the rates of influenza vaccination administered in physician's offices.

Pregnant women were stratified according to the presence or absence of any of the following pre-existing conditions (all information obtained from the NSAPD): pre-existing diabetes, pulmonary disease (including asthma), heart disease, renal disease, and anemia (hemoglobin < Io g) during the pregnancy. Women with I or more of these conditions were defined as having comorbidities. All other women were defined as having no comorbidities.

Trimesters were determined by the date of the birth and gestational age at the time of birth. Gestational age was assigned using last menstrual period, if known, or a clinical estimate of gestational age based on examination of the newborn. If both of these estimates of gestational age were missing, we imputed gestational age using the approach used

Table 1: Characteristics of study population

\begin{tabular}{|c|c|}
\hline Characteristic & $\begin{array}{c}\text { No. }(\%) \text { of women } \\
n=134188\end{array}$ \\
\hline \multicolumn{2}{|l|}{ Age at delivery, yr } \\
\hline$<20$ & 10633 \\
\hline $20-29$ & $74406(55.5)$ \\
\hline $30-34$ & $35207(26.2)$ \\
\hline $35-49$ & $13942(10.4)$ \\
\hline $\begin{array}{l}\text { Family income assistance } \\
\text { during birth year }\end{array}$ & $16154(12.0)$ \\
\hline Smoking during pregnancy & $38406(28.6)$ \\
\hline \multicolumn{2}{|l|}{ No. of children $<5 \mathrm{yr}$ of age } \\
\hline 0 & $82044(61.1)$ \\
\hline 1 & $44948(33.5)$ \\
\hline$\geq 2$ & $7196 \quad(5.4)$ \\
\hline Pre-existing diabetes & $539 \quad(0.4)$ \\
\hline Respiratory disease (including asthma) & $7416 \quad(5.5)$ \\
\hline Asthma & $6931 \quad(5.2)$ \\
\hline Heart disease & 1281 \\
\hline Renal disorder & $988 \quad(0.7)$ \\
\hline Anemia & 4051 \\
\hline Any high-risk comorbidity* & $13499(10.1)$ \\
\hline \multicolumn{2}{|l|}{ No. of comorbidities } \\
\hline 0 & 120689 (89.9) \\
\hline 1 & 12755 \\
\hline$>1$ & $744 \quad(0.6)$ \\
\hline
\end{tabular}

*Pre-existing diabetes, any respiratory disease, heart disease, renal disease or anemia. 
by Neuzil and colleagues ${ }^{8}$ (gestational age calculated as the median gestational age for infants born in the same birth year and whose birth weight was within the same 500-g category). Gestational age was imputed for less than $2 \%$ of the infants.

We based rates of hospital admissions on the date of admission minus 4 days to attribute the event to the influenza season during which most of the exposure occurred. To be conservative, admissions that resulted in a delivery and during which a respiratory illness was diagnosed were not counted in the rate, and only the first non-delivery-related hospital admission because of a respiratory illness was considered.

Person-time analyses were conducted for the entire pregnancy and by trimester. The number of events (hospital admissions or physician office visits) that occurred during the influenza, peri-influenza and non-influenza seasons was divided by the woman-months in each season to determine the event rate for each of the 3 defined seasons. For the trimesterspecific analyses, the number of events and woman-months were further divided into the amount of time a woman spent in each influenza season during each trimester. Poisson regression models were used to adjust for confounding factors, and rate ratios (and 95\% confidence intervals [CIs]) were calculated. Potential confounders included maternal age, maternal smoking status, socioeconomic status (based on receipt of family benefits), and number and ages of other children in the home. Since the outcomes for women with more than one pregnancy during the study period are not independent, generalized estimating equations were used to generate unbiased standard errors and $95 \%$ CIs. ${ }^{12}$

Initially, we calculated unadjusted rate ratios. The potential confounding factors were entered into the model, with season as the independent variable of interest. Each potential confounding factor was removed, one at a time, and the model rerun. If removing the factor did not change the coefficient (for season) by $5 \%$ or more, the factor was removed and the process repeated. A change of $5 \%$ or more was chosen so that factors would be included in the final model if they produced a modest change in the coefficient for season. With this approach, the final model included factors that confounded the relation between season and rates of hospital admission.

Rates of hospital admissions (and physician office visits) during the influenza, peri-influenza and non-influenza seasons for each trimester of pregnancy were compared with rates of admissions (and physician visits) during these 3 seasons in the year before pregnancy for the same cohort of women. In addition, rates of hospital admissions (and physician visits) for each trimester of pregnancy during the influenza and peri-influenza seasons were compared with rates for each trimester during the non-influenza season.

Influenza-attributable risks in each trimester of pregnancy were estimated by subtracting the rate of hospital admissions during the peri-influenza and non-influenza seasons from the rate during the influenza season, as described by Neuzil and colleagues. ${ }^{8}$ We used the rates of hospital admissions during the peri-influenza season to determine the baseline risk to help quantify the risks specific to influenza-related complications rather than to complications that may be related to other viruses (e.g., respiratory syncytial virus).

This study received approval from the Research Ethics Board of the IWK Health Centre.

\section{Results}

There were I34 I88 pregnant women in the study cohort. Overall, I3 499 (I0.I\%) of these women had a medical comorbidity that put them at increased risk of influenza-associated morbidity; of them, $94.5 \%$ had just I high-risk condition (Table I). Asthma and anemia were the most common conditions, present in $5.2 \%$ and $3.0 \%$ of the women respectively. A total of 510 women $(0.4 \%)$ were admitted to hospital during pregnancy because of a respiratory illness (during any season), and 33775 (25.2\%) visited their physician at least once during pregnancy for the same reason (during any season).

For women with and without comorbidities, the rate ratios of hospital admissions during the influenza season were higher in all trimesters of pregnancy than in the year before pregnancy (Table 2). This difference was most evident among pregnant women with comorbidities, in whom there was an excess of 39.2 hospital admissions per to ooo woman-months in the third trimester compared with the year before pregnancy (rate ratio 7.9; $95 \%$ CI 5.0-12.5). Even among women without comorbidities, there was an excess of 6.0 hospital admissions in the third

Table 2: Hospital admissions because of respiratory illness during the influenza season in the year before pregnancy and during pregnancy, by presence of comorbidities

\begin{tabular}{|c|c|c|c|c|c|c|}
\hline \multirow[b]{2}{*}{ Period } & \multicolumn{3}{|c|}{ Women with no comorbidity } & \multicolumn{3}{|c|}{ Women with $\geq 1$ comorbidity } \\
\hline & $\begin{array}{l}\text { No. of admissions } \\
\text { during influenza } \\
\text { season }\end{array}$ & $\begin{array}{l}\text { Rate per } 10000 \\
\text { woman-months }\end{array}$ & $\begin{array}{l}\text { Rate ratio } \\
(95 \% \mathrm{Cl})^{*}\end{array}$ & $\begin{array}{l}\text { No. of admissions } \\
\text { during influenza } \\
\text { season }\end{array}$ & $\begin{array}{l}\text { Rate per } 10000 \\
\text { woman-months }\end{array}$ & $\begin{array}{l}\text { Rate ratio } \\
(95 \% \mathrm{Cl})^{*}\end{array}$ \\
\hline Year before pregnancy & 49 & 1.4 & 1.0 & 23 & 5.7 & 1.0 \\
\hline \multicolumn{7}{|l|}{ Pregnancy } \\
\hline Second trimester & 30 & 3.0 & $2.1(1.3-3.3)$ & 22 & 19.4 & $3.4(1.9-6.0)$ \\
\hline Third trimester & 76 & 7.4 & $5.1(3.6-7.3)$ & 49 & 44.9 & $7.9(5.0-12.5)$ \\
\hline
\end{tabular}

Note: $\mathrm{Cl}=$ confidence interval

*Rate ratio of admissions during pregnancy compared with admissions in the year before pregnancy. 
trimester per Io ooo woman-months during the influenza season compared with the admissions during the influenza season in the year before pregnancy (rate ratio 5.I; 95\% CI 3.6-7.3). Adjustment for potential confounders, including socioeconomic status, did not change the rate ratio estimates, and these confounders were therefore not included in the final model.

When we compared rates of hospital admissions by trimester between the influenza and non-influenza seasons, we observed higher rates during the influenza season among both women with and those without comorbidities in all trimesters (Table 3). The rate of hospital admissions was highest in the third trimester, particularly among women with comorbidities. The rate ratio of admissions in the third trimester during the influenza season compared with third- trimester admissions during the non-influenza season was 2.4 among women with comorbidities (95\% CI I.6-3.6); the rate ratio was similarly increased among women without comorbidities (rate ratio 2.4; 95\% CI I.7-3.4).

The rates of hospital admissions increased as pregnancy progressed, even when no influenza activity was detected in the community (Table 3). This increase occurred predominantly among women with comorbidities, among whom the rates of admissions during the non-influenza season were on average 6fold higher than those among women without comorbidities.

Among women without comorbidities, the rate of physician office visits during the influenza season did not differ significantly during pregnancy from the rate in the year before pregnancy (Table 4). However, among women with co-

Table 3: Hospital admissions because of respiratory illness in influenza and non-influenza seasons, by trimester and presence of comorbidities

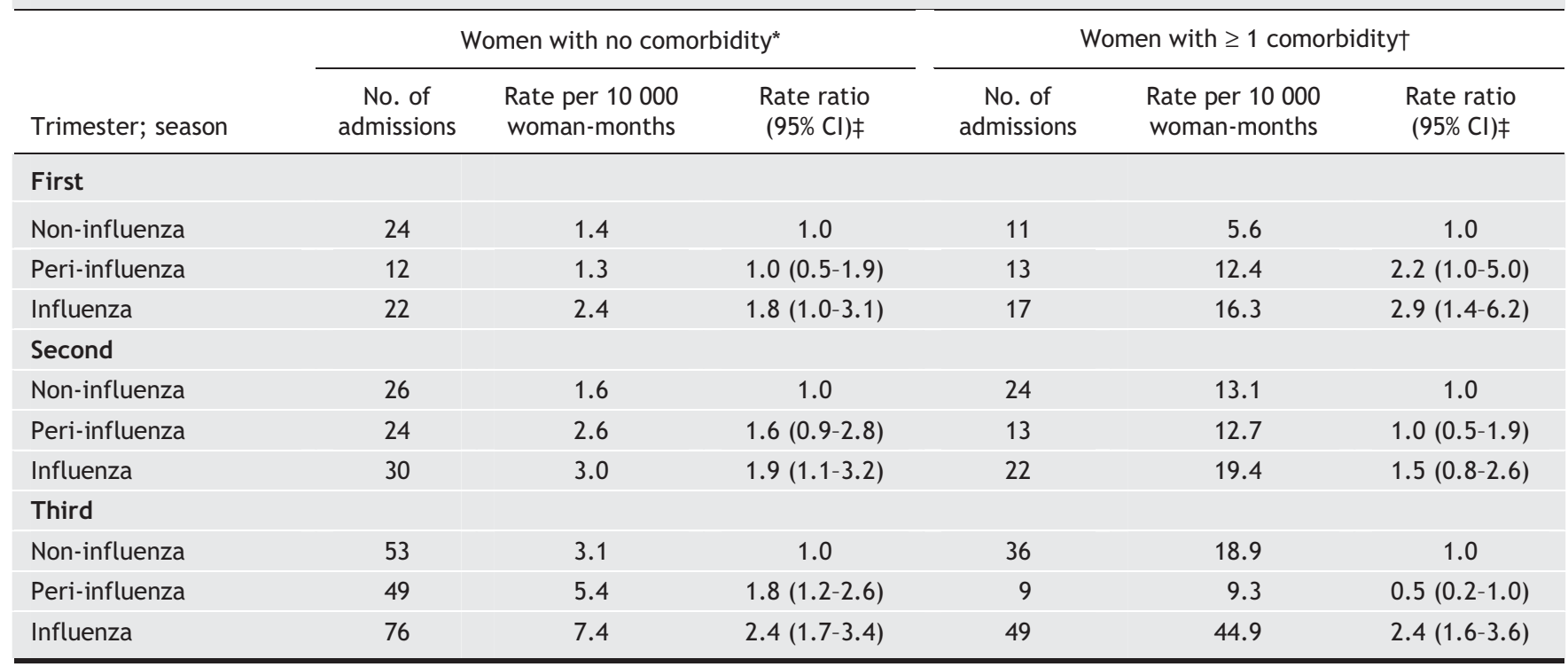

Note: $\mathrm{Cl}=$ confidence interval.

*Among women with no comorbidities, the attributable differences in rates (ARs) between influenza season and peri-influenza season were 1.1 in the first trimester, 0.4 in the second trimester and 2.0 in the third trimester. The corresponding ARs between influenza season and non-influenza season were $1.0,1.4$ and 4.3 .

†Among women with $\geq 1$ comorbidity, the ARs between influenza season and peri-influenza season were 3.9 in the first trimester, 6.7 in the second trimester and 35.6

in the third trimester. The corresponding ARs between influenza season and non-influenza season were 10.7, 6.3 and 26.0.

¥Rate ratio of trimester-specific admissions during influenza and peri-influenza seasons compared with admissions during non-influenza season.

Table 4: Physician visits because of respiratory illness during the influenza season in the year before pregnancy and during pregnancy, by presence of comorbidities

\begin{tabular}{|c|c|c|c|c|c|c|}
\hline \multirow[b]{2}{*}{ Period } & \multicolumn{3}{|c|}{ Women with no comorbidity } & \multicolumn{3}{|c|}{ Women with $\geq 1$ comorbidity } \\
\hline & $\begin{array}{l}\text { No. of visits during } \\
\text { influenza season** }\end{array}$ & $\begin{array}{l}\text { Rate per } 10000 \\
\text { woman-months }\end{array}$ & $\begin{array}{c}\text { Rate ratio } \\
(95 \% \mathrm{Cl}) \dagger\end{array}$ & $\begin{array}{l}\text { No. of visits during } \\
\text { influenza season* }\end{array}$ & $\begin{array}{l}\text { Rate per } 10000 \\
\text { woman-months }\end{array}$ & $\begin{array}{l}\text { Rate ratio } \\
(95 \% \mathrm{Cl}) \dagger\end{array}$ \\
\hline Year before pregnancy & 17560 & 512.6 & 1.0 & 2913 & 720.0 & 1.0 \\
\hline \multicolumn{7}{|l|}{ Pregnancy } \\
\hline First trimester & 4490 & 486.7 & $0.9(0.9-1.0)$ & 823 & 788.5 & $1.1(1.0-1.2)$ \\
\hline Third trimester & 4889 & 473.2 & $0.9(0.9-1.0)$ & 951 & 871.1 & $1.2(1.1-1.4)$ \\
\hline
\end{tabular}

Note: $\mathrm{Cl}=$ confidence interval

*Some women visited their physician more than once.

†Rate ratio of physician visits during pregnancy compared with visits in the year before pregnancy. 
morbidities, the rate of physician visits during the influenza season was $20 \%$ higher in the third trimester than in the year before pregnancy. Compared with rates in the non-influenza and peri-influenza seasons, rates of physician visits were higher during the influenza season in all trimesters among women with and without comorbidities (data not shown).

During the years for which immunization data were available, we observed that $2.6 \%$ of the women in the study cohort and $6.7 \%$ of those with a comorbidity had received an influenza vaccine during pregnancy. These data represent immunizations in physicians' offices and therefore may underrepresent the number of women who were immunized.

\section{Interpretation}

This study provides robust, population-based data showing that women at all stages of pregnancy are at increased risk of serious respiratory illness during the influenza season, even in the absence of pre-existing comorbid conditions known to increase the risk of influenza-associated morbidity. Among the women in our cohort who had no comorbidities, the rate of third-trimester hospital admissions during the influenza season was 5 times higher than the rate during the influenza season in the year before pregnancy and more than twice as high as the rate during the non-influenza season. Among the women with comorbidities, the risk of respiratory illness during the influenza season increased markedly as pregnancy progressed but was substantial in all trimesters. In the third trimester, the excess of hospital admissions observed during the influenza season compared with admissions during the peri-influenza season was 35.6 per io ooo woman-months among women with comorbidities and 2.0 per to ooo womanmonths among women without comorbidities. Given that the influenza season in Nova Scotia lasted 3.4 months on average over the study period, this represents an excess of I2Io hospital admissions in the third trimester per 100 ooo pregnant women with comorbidities and 68 per 100 ooo without comorbidities.

To put our data into context, the rate of excess hospital admissions that we observed during the influenza season among healthy pregnant women in their third trimester (68 per 100 000) is lower than that reported among healthy children less than 2 years of age (90-I038 per 100 000 $)^{13-16}$ but is comparable to observed rates among American adults aged I5-44 years with comorbid conditions (56-IIo per 100 000), ${ }^{17}$ a group for whom annual influenza immunization is already recommended. ${ }^{1,2,18,19}$

Although our study involved women from a single Canadian province, the sample was large and incorporated a cohort of pregnant women over a study period of 13 years. This population is similar to the general Canadian population of pregnant women and that of pregnant women in other industrialized nations and is more representative than the Tennessee Medicaid population in the study by Neuzil and colleaegues. ${ }^{8}$

Patterns of hospital admission rates in our study were similar to those reported by Neuzil and colleagues; ${ }^{8}$ however, the actual rates were considerably lower in our study, among both women with and those without comorbidities, during all trimesters of pregnancy and among women in the year before they were pregnant. The proportion of women with comorbidities was similar in both studies (about 10\%), with chronic pulmonary disease being the most prevalent risk factor in both populations. The observed differences in hospital admission rates between the 2 studies may have been due in part to differences in the ascertainment of study outcomes. To be conservative, we excluded hospital admissions that resulted in delivery as well as admissions because of exacerbated asthma in which no influenza-specific ICD code was identified. Dissimilarity in access to care may also have contributed to the differences in admission rates, since income distribution and universal access to publicly funded health care are the most striking differences between our study population and the Medicaid population studied by Neuzil and colleagues. Populations with an unequal distribution of income are known to experience worse health outcomes and increased mortality than populations in which income distribution is more homogeneous. ${ }^{20-27}$ Adjustment for family income support did not alter the magnitude of the rate ratios observed in our study.

Inactivated influenza vaccine has a long track record of use in pregnant women and is considered safe in all stages of pregnancy. ${ }^{1,2,28-34}$ Antibody responses to influenza vaccine have been reported to be similar among pregnant and nonpregnant women. ${ }^{29,30,34}$ However, vaccine effectiveness has been more difficult to demonstrate because of low immunization rates among pregnant women..$^{8,28-35}$ In our study, only $2.6 \%$ of all pregnant women and $6.7 \%$ of pregnant women with a comorbid condition were immunized.

In conclusion, our study provides robust, populationbased data showing that all pregnant women are at increased risk of influenza-associated respiratory illness. Concerted efforts should be made to ensure communication of the risks of influenza during pregnancy to women and their health care providers to ensure that all pregnant women have the opportunity to benefit from influenza immunization. Future studies should examine the cost-effectiveness of implementing a publicly funded influenza immunization program for all pregnant women and should include costs associated with hospital admissions and outpatient physician visits as well as broader societal costs.

\section{This article has been peer reviewed.}

From the Canadian Centre for Vaccinology (Dodds, McNeil, Allen, Coombs, Scott, MacDonald); the Perinatal Epidemiology Research Unit, IWK Health Centre (Dodds, Fell); the Departments of Obstetrics and Gynaecology (Dodds, Allen), Pediatrics (Dodds, McNeil, MacDonald) and Medicine (McNeil), Dalhousie University; and the Nova Scotia Department of Health (Coombs, Scott), Halifax, NS

Competing interests: None declared for Linda Dodds, Deshayne Fell, Victoria Allen and Ann Coombs. Shelly McNeil is a member of the Canadian National Advisory Committee on Immunization. At the time of writing, Noni MacDonald was Acting Editor-in-Chief of CMAJ, and Jeffrey Scott was a member of the Interim Editorial Board of CMAJ.

Contributors: Linda Dodds, Shelly McNeil and Noni MacDonald conceived the project and developed the protocol. Linda Dodds and Shelly McNeil were the principal investigators for the study and wrote the first draft of the manuscript. Deshayne Fell contributed to the development of the analysis plan and analyzed the data. Victoria Allen contributed expertise in maternal and fetal outcomes and assisted in the development of the protocol and data analysis. Ann Coombs and Jeffrey Scott contributed expertise in determining influenza 
seasons and assisted in the development of the protocol and the interpretation of data. Noni MacDonald contributed to the development of the protocol and analysis plan, and the interpretation and dissemination of data. All of the authors helped draft the manuscript and approved the version to be published.

Acknowledgements: We thank the Reproductive Care Program of Nova Scotia and the Population Health Research Unit at Dalhousie University for facilitating access to the data.

This study was funded by a grant from the Canadian Institutes of Health Research. Linda Dodds, Shelly McNeil and Victoria Allen are supported by Clinical Research Scholar Awards from Dalhousie University and Dodds and Allen are supported by New Investigator Awards of the Canadian Institutes of Health Research. Although this research is based in part on data obtained from the Population Health Research Unit, the observations and opinions expressed are those of the authors and do not represent those of the Population Health Research Unit.

\section{REFERENCES}

I. Orr P, National Advisory Committee on Immunization. Statement on influenza vaccination for the 2004-2005 season. Can Commun Dis Rep 2004;30ACS-3:I-32.

2. Harper SA, Fukuda K, Uyeki TM, et al. Prevention and control of influenza: recommendations of the Advisory Committee on Immunization Practices (ACIP) [published erratum in MMWR Recomm Rep 2004;53(32):743]. MMWR Recomm Rep 2004;53(RR-6):I-40.

3. Harris JW. Influenza occurring in pregnant women. JAMA 1919;72:978-80.

4. Freeman DW, Barno A. Deaths from Asian influenza associated with pregnancy. Am J Obstet Gynecol 1959;78:1172-5.

5. Kort BA, Cefalo RC, Baker VV. Fatal influenza A pneumonia in pregnancy. Am J Perinatol $1986 ; 3: 179-82$.

6. Kirshon B, Faro S, Zurawin RK, et al. Favourable outcome after amantadine and ribaviron in a pregnancy complicated by influenza pneumonia. J Reprod Med I988; 33:399-40I.

7. Schoenbaum SC, Weinstein L. Respiratory infection in pregnancy. Clin Obstet Gynecol 1979;22:293-300.

8. Neuzil KM, Reed GW, Mitchel EF, et al. Impact of influenza on acute cardipulmonary hospitalizations in pregnant women. Am J Epidemiol I998;I48:I094-I02.

9. MacDonald NE, McNeil SA, Allen VM, et al. Influenza vaccine programs and pregnancy: A need for more evidence. J Obstet Gynaecol Can 2004;26:96I-3.

Io. Hartert TV, Neuzil KM, Shintani AK, et al. Maternal morbidity and perinatal outcomes among pregnant women with respiratory hospitalizations during influenza season. Am J Obstet Gynecol 2003;198:1705-I2.

II. Fair M, Cyr M, Allen AC, et al. Validation study for a record linkage of births and infant deaths in Canada. Ottawa: Statistics Canada;I999. Cat. no. 84FoorXIE. Available: www.statcan.ca/english/research/84Foor 3 XIE/84Foor 3 XIEI9920or.pdf (accessed 2007 Jan 3).

I2. Louis GB, Dukic V, Heagerty PJ, et al. Pregnancy Modeling Working Group. Analysis of repeated pregnancy outcomes. Stat Methods Med Res 2006;15:103-26.

13. Neuzil KM, Mellen BG, Wright PF, et al. Effect of influenza on hospitalizations, outpatient visits, and courses of antibiotics in children. N Engl J Med 2000;342: 225-3I.

I4. Izurieta HS, Thompson WW, Kramarz P, et al. Influenza and the rates of hospitalization for respiratory disease among infants and young children. $N$ Engl J Med 2000;342:232-9.

15. Neuzil KM, Zhu Y, Griffin MR, et al. Burden of interpandemic influenza in children younger than 5 years: a 25-year prospective study. JInfect Dis 2002;185:147-52.
I6. Griffin MR, Walker FJ, Iwane MK, et al. New Vaccine Surveillance Network Study Group. Epidemiology of respiratory diseases in young children. Insights from the new vaccine surveillance network. Pediatr Infect Dis J 2004;23:SI88-92.

I7. Barker WH, Mullooly JP. Impact of epidemic type A influenza in a defined adult population. Am J Epidemiol I980;112:798-8II.

I8. Australian Government Department of Health and Ageing. National Health and Medical Research Council recommendations on influenza vaccination. Available: www.health.gov.au/internet/wcms/publishing.nsf/content/cda-surveil-ozflu-flunhmrc .htm (accessed 2007 Jan 3).

I9. Salisbury D, Ramsay M, Noakes K, editors. Immunisation against infectious disease. 3 rd ed. London (UK): The Stationary Office; 2006. Available: www .dh.gov.uk/PolicyAndGuidance/HealthAndSocialCareTopics/GreenBook /GreenBookGeneralInformation/GreenBookGeneralArticle/fs/en?CONTENT _ID $=4097254 \&$ chk=isTfGX (accessed 2007 Jan 3).

20. Ben-Shlomo Y, White IR, Marmot M. Does the variation in the socioeconomic characteristics of an area affect mortality? BMJ I996;312:I0I3-4.

21. Fiscella K, Franks P. Poverty or income inequality as predictors of mortality: longitudinal cohort study. BMJ I997;314:1724-7.

22. Flegg A. Inequality of income, illiteracy, and medical care as determinants of infant mortality in developing countries. Popul Stud (Camb) I982;36:44I-58.

23. Kaplan GA, Pamuk ER, Lynch JW, et al. Income inequality and mortality in the United States: analysis of mortality and potential pathways. BMJ I996;312:999-1003.

24. Kennedy BP, Kawachi I, Prothrow-Stith D. Income distribution and mortality: cross-sectional ecological study of the Robin Hood index in the United States. BMJ I996;312:1004-7

25. Lynch JW, Kaplan GA, Pamuk ER, et al. Income inequality and mortality in metropolitan areas of the United States. Am J Public Health 1998;88:1074-80.

26. Wilkinson RG. Income distribution and life expectancy. BMJ I992;304:I65-8.

27. Ross NA, Wolfson MC, Dunn JR, et al. Relation between income inequality and mortality in Canada and in the United States: cross-sectional assessment using census data and vital statistics. BMJ 2000;320:898-902.

28. Heinonen OP, Slone D, Shapiro S. Immunizing agents. In: Kaufman DW, editor. Birth defects and drugs in pregnancy. Littleton (MA): Publishing Sciences Group; I977. p. 3I4-2I.

29. Sumaya CV, Gibbs RS. Immunization of pregnant women with influenza A/New Jersey/ 76 virus vaccine: reactogenicity and immunogenicity in mother and infant. $J$ Infect Dis I979;I40:I4I-6.

30. Murray DL, Imagawa DT, Okada DM, et al. Antibody response to monovalent A/New Jersey/8/76 influenza vaccine in pregnant women. JClin Microbiol I979;10:184-7.

31. Deinard AS, Ogburn P Jr. A/NJ/8/76 influenza vaccination program: effects on maternal health and pregnancy outcome. Am J Obstet Gynecol I981;140:240-5.

32. Englund JA, Mbawuike IN, Hammill H, et al. Maternal immunization with influenza or tetanus toxoid vaccine for passive antibody protection in young infants. Jnfect Dis 1993; 168:647-56.

33. Munoz FM, Greisinger AJ, Wehmanen OA, et al. Safety of influenza vaccination during pregnancy. Am J Obstet Gynecol 2005;192:1098-Io6.

34. La Montagne JR, Noble GR, Quinnan GV, et al. Summary of the clinical trials of inactivated influenza vaccine-I978. Rev Infect Dis I983;5:723-36.

35. Black SB, Shinefield HR, France EK, et al. Vaccine Safety Datalink Workgroup. Effectiveness of influenza vaccine during pregnancy in preventing hospitalizations and outpatient visits for respiratory illness in pregnant women and their infants. Am J Perinatol 2004;21:333-9.

Correspondence to: Dr. Linda Dodds, Departments of Obstetrics and Gynaecology and of Pediatrics, Perinatal Epidemiology Research Unit, IWK Health Centre, $5850 / 5980$ University Ave., PO Box 9700, Halifax NS B3K 6R8; l.dodds@dal.ca

\section{LEADERSHIP}

$C M A J$ is a founding member of the International Committee of Medical Journal Editors, an organization that is devoted to ensuring the highest integrity in scientific publishing and is a driving force in the mandatory registration of clinical trials. 